\title{
Lifing the thermo-mechanical fatigue (TMF) behaviour of the polycrystalline nickel-based superalloy RR1000
}

\author{
Jonathan Jones $^{1}$, Mark Whittaker ${ }^{1}$, Robert Lancaster ${ }^{1}$, and Stephen Williams ${ }^{2}$ \\ ${ }^{1}$ Swansea University, Singleton Park, Swansea SA2 8PP, UK \\ 2 Rolls-Royce, PO Box 31, Derby DE24 8BJ, UK
}

\begin{abstract}
Microstructural damage and subsequent failures resulting from thermo-mechanical fatigue (TMF) loading within the temperature range $300-700^{\circ} \mathrm{C}$ are investigated for the polycrystalline nickel superalloy, RR1000. Strain controlled TMF experiments were conducted over various mechanical strain ranges, encompassing assorted phase angles, using hollow cylindrical test pieces. The paper explores two scenarios; the first where the mechanical strain range is held constant and comparisons of the fatigue life are made for different phase angle tests, and secondly, the difference between the behaviour of In-phase (IP) and $-180^{\circ}$ OutOf-Phase (OOP) tests over a variety of applied strain ranges. It is shown that different lifing approaches are currently required for the two scenarios, with a mean stress based approach being more applicable in the first case, whereas a Basquin-type model proves more appropriate in the second.
\end{abstract}

\section{Introduction}

During the take-off, cruise, descent and landing cycle that jet engines experience when in service, high temperature features such as disc rims typically endure severe alternating thermal and mechanical loads. Under such situations, cracks can potentially nucleate and propagate to failure, through a phenomenon which is known as ThermoMechanical Fatigue (TMF). It is widely acknowledged to be one of the most significant damage mechanisms facing the gas turbine sector as designers push materials closer and closer to their operating limits, striving to meet the demanding emissions and fuel burn ACARE "Flightpath 2050" targets. Increasing operating temperatures to improve cycle efficiency, in conjunction with weight reduction strategies that include thinner disc rims, have led not only to more aggressive TMF cycles, but also to the requirement for assessment of the behaviour of components for which TMF was not previously considered significant.

Typically, several material properties including creep and fatigue are used to assess design lifetimes of high temperature components such as turbine discs. However, many of these mechanical properties are obtained from isothermal tests as testing under TMF conditions is difficult. It is an expensive and time consuming test method with a wide range of variables that require consideration such as peak cycle temperature, temperature range, applied strain range, $\mathrm{R}$ ratio, phase angle (between thermal and mechanical strains), waveform and applied strain rate. Accordingly, a data generation programme that encompasses all of these TMF factors is expensive and unrealistic. Therefore there is a clear requirement for a robust predictive model which can provide accurate extrapolation from a known set of results. Furthermore, it is desirable that the model, if possible, utilises isothermal data in order to further simplify the input data requirements. However, this is obviously a significant extension of models which predict only isothermal data and it is necessary to investigate whether such models are compromised by the damage mechanisms involved in TMF.

This paper considers a number of lifing approaches to determine the effects of phase angle on TMF life in the polycrystalline nickel alloy RR1000. RR1000 is produced through a powder metallurgy process and is widely anticipated to play a significant role in upcoming engines as a High Pressure (HP) disc rotor material. However, studies into the TMF behaviour of the alloy have been extremely limited and TMF lifing of many alloys tends to follow the approach of attempting extrapolation from isothermal data and particularly assuming isothermal data at the peak cycle temperature to be a worst case scenario. Within the gas turbine, however, this approach may be extremely limited due to the wide range of phase angles possible and the marked effect of a TMF cycle.

The phase angle as defined for a TMF test is the shift which occurs between the mechanical strain and the temperature applied to the material. TMF typically operates in one of two ways; either under an In-Phase (IP) condition, where the maximum loading on the material coincides with the maximum temperature, or an Outof-Phase (OP) situation $\left(-180^{\circ}\right)$, where the maximum loading occurs at the lowest temperature. However, at present few investigations have looked into the alternative phase angles which could be applied between these two extremes and furthermore an added complication is that the cycle can take one of two directions around the straintemperature loop, either clockwise $(\mathrm{CW})$ or anticlockwise $(\mathrm{ACW})$, as shown in Fig. 1.

As such it is critical that any model which is sought to describe TMF behaviour is capable of interpolation 


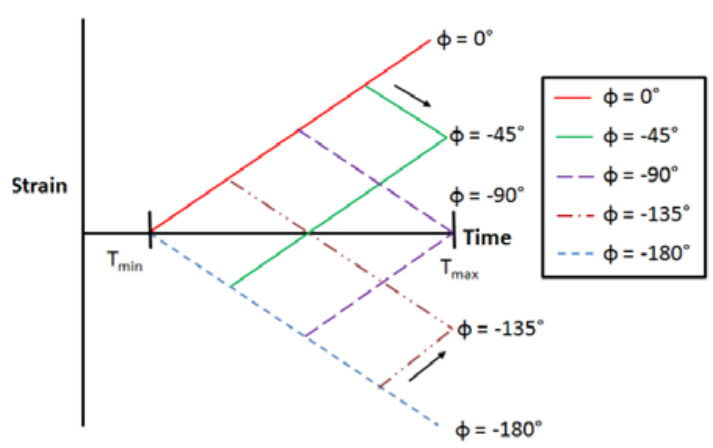

Figure 1. Phase angles $(\phi)$ and directions.

across phase angles, and if at all possible different maximum/minimum temperatures, in order to allow designers the capacity for accurate life prediction of critical parts.

Previous attempts to model TMF behaviour in the literature [1-4], especially in nickel based superalloys for disc applications, are quite limited. They do however, fall broadly into two categories; the first assumes that under strain controlled loading a significant fraction of the TMF life is spent in crack initiation and as such traditional approaches such as strain-energy partitioning, MansonCoffin [5] etc., can be applied [6,7]. Conversely, the second approach assumes that cracks form very early in the test, possibly even on the first cycle [8-10]. On this basis the fatigue life of the specimen will be dominated by crack propagation. Each type of approach has been shown to have its own benefits and drawbacks and it is also clear that the most appropriate method will be material and temperature dependent.

\section{Material and specimen design}

The $\gamma^{\prime}$ strengthened polycrystalline Ni-based superalloy, RR1000, was used in this investigation, with its chemical composition displayed in Table 1 . The alloy is produced through a powder metallurgy route and strengthened through the precipitation of a secondary $\gamma^{\prime}$ phase $\left(\mathrm{Ni}_{3}(\mathrm{Al}\right.$, $\mathrm{Ti}, \mathrm{Ta})$ ) from an FCC $\gamma$ matrix. The desired average $\gamma$ grain size of 4-8 $\mu \mathrm{m}$ was achieved through a sub-solvus solution heat treatment, leaving irregular shaped primary $\gamma^{\prime}$ particles (1-5 $\mu \mathrm{m}$ in size) on grain boundaries. A typical scanning electron microscope image of the microstructure of FG RR1000 is given in Fig. 2.

Tubular hollow test specimens of nominal external diameter $10 \mathrm{~mm}$, internal diameter $8 \mathrm{~mm}$ and a parallel gauge length of $25 \mathrm{~mm}$ were utilised for testing. The temperature distribution across the test specimen was shown to conform to the guidelines defined in ISO 12111:2011 [11], i.e. the linear temperature distribution within the extensometer arms remains less than $\pm 10^{\circ} \mathrm{C}$ at peak temperature and $\pm 6^{\circ} \mathrm{C}$ at minimum temperature, and the radial temperature distribution remains within $5^{\circ} \mathrm{C}$ of the required temperature. According to the TMF Code of Practice (TMF COP) [12], standard pre-requisite tests including modulus checks, thermal compensation definitions and zero stress trials were also performed to ensure test viability.

\section{Experimental procedure}

All TMF tests were performed on an ESH $100 \mathrm{KN}$ tension-torsion servo-hydraulic test machine under straincontrolled conditions. Extension was controlled/ measured using a $12 \mathrm{~mm}$ gauge MTS high temperature extensometer with the total strain and thermal cycles synchronised and controlled by a closed loop computer to facilitate the phasing.

Thermal cycling was applied via a water cooled copper radio frequency induction coil, with internal and external cooling jets used to aid the demanding cooling rate on the specimen. The temperature was measured by an IMPAC IP10 optical pyrometer which was connected to a two way control system.

Preliminary verification examinations determined that the temperature difference across the extensometer gauge length did not deviate by more than $\pm 2 \%$ of the desired temperature, or $\pm 10^{\circ} \mathrm{C}$, whichever was the least, in both a static and dynamic situation. These conditions were consistent with those defined in the TMF Code-of-Practice (COP) and previous work $[13,14]$. A linear thermal cycle thought to be representative of a typical in-service TMF cycle for a turbine disc of $300-700-300^{\circ} \mathrm{C}$ was used throughout testing. The thermal cycle has a ramp time of 15 seconds, producing a 30 second cycle triangular temperature waveform.

Concerns have previously been raised regarding the use of pyrometers during such high temperature applications, since the formation of oxidation at high temperature is prevalent, causing changes in the material's surface emissivity. The emissivity of the material was characterised as a function of time and temperature to overcome these concerns. It was shown that at $700{ }^{\circ} \mathrm{C}$ the emissivity of RR1000 reaches a stable value after 30 hours, with oxidation damage under these conditions expected to be $<1 \mu \mathrm{m}$. As such, specimens were pre-oxidised in a furnace for this period of time to create a stable oxide layer prior to the commencement of any TMF experiments. No further changes in emissivity were noted, and subsequent temperature control was accurately achieved throughout the test.

In order to evaluate the effect of phase angle on the TMF life of RR1000, tests were performed at $\phi=0,-45$ $(\mathrm{CW}),-90(\mathrm{CW}+\mathrm{ACW}),-135(\mathrm{ACW})$, and $-180^{\circ}$ as shown in Fig. 1. Tests were performed using a constant mechanical strain range of $1 \%$, resulting in strain $\mathrm{R}$ ratios varying between 0 and $-\infty$ depending on the phase angle $\phi$. Further tests were performed under IP and $-180^{\circ}$ OOP loading conditions in which the applied mechanical strain range was varied between 0.8 and $1.4 \%$ in order to investigate the effect of this property on TMF life. Failure was defined as the number of cycles to achieve a $10 \%$ drop in stress from the stabilised peak stress value at which point a detectable engineering sized crack is assumed to be present in the sample.

\section{Results}

In order to derive a model that accurately describes TMF behaviour, it is of course critical that the data produced by the experiments are of the highest quality possible. 
Table 1. Chemical composition of RR1000 (wt.\%).

\begin{tabular}{|l|l|l|l|l|l|l|l|l|l|l|}
\hline Co & Cr & Mo & Ti & Al & Ta & Hf & C & B & Zr & Ni \\
\hline 18.5 & 15 & 5 & 3.6 & 3 & 2 & 0.5 & 0.027 & 0.015 & 0.055 & Bal \\
\hline
\end{tabular}

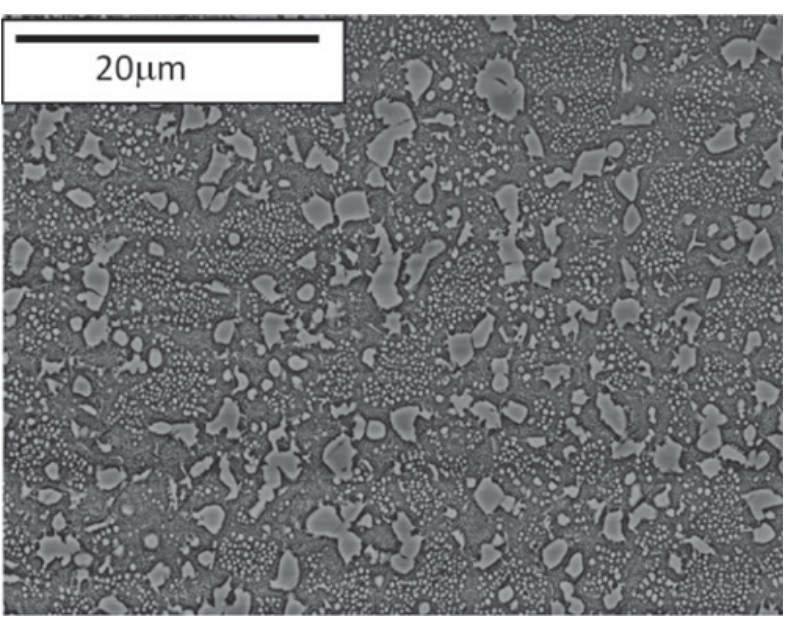

Figure 2. Microstructure of FG RR1000.

In the case of the current work the quality of the test data can be easily determined from the appearance of the mechanical strain-stress hysteresis loops. During the current experiments the data returned appear to be of a high quality, allowing for consistent interpretation. Figure 3 provides examples of the loops obtained from both IP and $-180^{\circ}$ OOP tests with strain ranges of $1.4 \%$. As would be expected in TMF loops, yield is not particularly well defined due to the changing modulus values as temperature increases in the initial part of the cycle. An increase in the absolute value of the compressive stress can also be seen to occur between the first and stabilised loading cycles (Fig. 3a). Similar effects can also be observed in compression in the $-180^{\circ}$ OOP loop (Fig. 3b). With the quality of the stress-strain loops established, confidence can be placed in the corresponding fatigue lives. As such, in order to relate isothermal behaviour to TMF, the lives of the tests are normalised by the fatigue life of an isothermal fatigue test on the material at $\mathrm{R}=0, \Delta \varepsilon=1 \%$ at peak cycle temperature $\left(700^{\circ} \mathrm{C}\right)$, and therefore a positive or negative effect of the TMF cycle on life can be easily demonstrated.

Essentially, two studies were undertaken as described previously. In Fig. 4 tests are conducted at a range of phase angles as described by Fig. 1, whilst maintaining a constant applied mechanical strain range, $\Delta \varepsilon=1 \%$. The conditions of these tests offer an interesting opportunity for comparison, since although the $\mathrm{R}$ ratio varies from test to test, the selected phase angles provide identical straintemperature paths for portions of each cycle. It is clear that phase angle significantly affects TMF life, with a gradual decrease in life as the phase angle increases from $-180^{\circ}$ to $0^{\circ}$, as shown in Fig. 4.

\section{Discussion}

Based on the wide range of variables which occur during a TMF test it is clear that a lifing approach capable of holistic

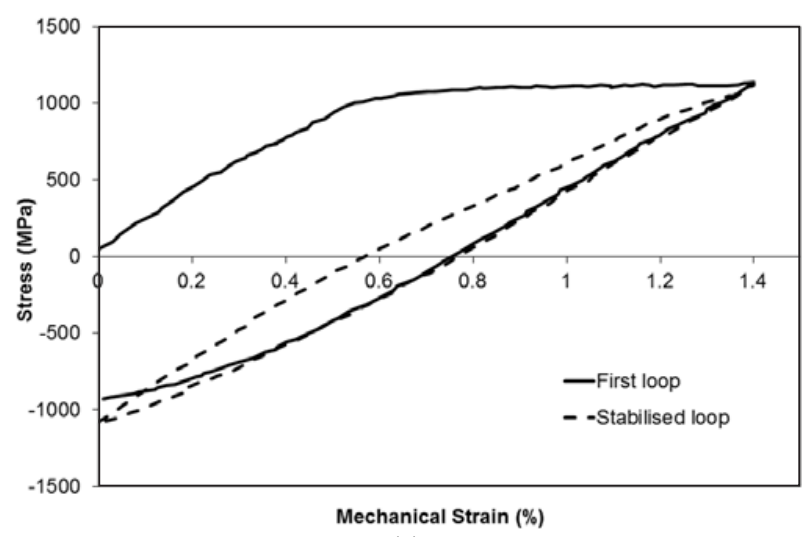

(a)



(b)

Figure 3. $1^{\text {st }}$ and stabilised stress-strain hysteresis loops for (a) IP test, $\Delta \varepsilon=1.4 \%$ (b) OOP test, $\Delta \varepsilon=1.4 \%$.

data fitting may not be possible. However, an objective of the current work is to assess possible lifing strategies in order to provide a basis for a more widespread approach. As such, the discussion will focus on the advantages and drawbacks of methods based around mean stress, stabilised stress range and crack propagation life. Based on the results produced within this work, no difference was seen between specimens loaded in a clockwise (CW) or anticlockwise (ACW) direction around the straintemperature loop for a $90^{\circ}$ (OOP) phase angle. As such the approaches here are simplified by not considering directionality in these tests.

One relatively straightforward approach to ranking TMF results can be made by simply considering the stabilised mean stress achieved during the test, with the expectation being that an increased mean stress for a constant stress range will lead to a reduction in fatigue life. Due to the non-isothermal nature of the TMF test, stressstrain hysteresis loops can vary dramatically in shape for different phase angles, leading to significant changes in mean stress. The most common cause of this is the alternating contributions between creep strain and plasticity, typical in the TMF cycle. By considering an IP cycle, in 


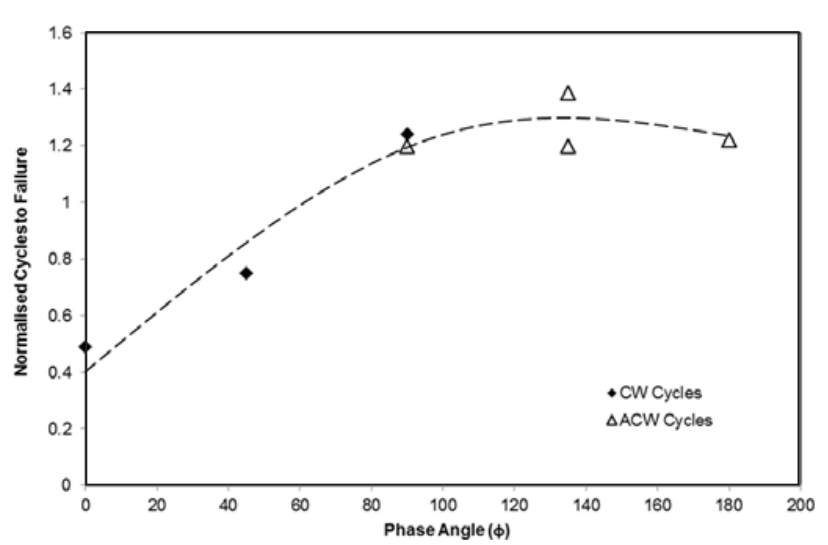

Figure 4. Phase angle $(\phi)$ vs. number of TMF cycles to failure $\left(\mathrm{N}_{\mathrm{f}}\right)$ for constant strain range $(\Delta \varepsilon)$ of $1 \%$.

which the strain and temperature increase in proportion, it can be appreciated that yield will occur within the material and dislocations will be generated within the grains, resulting in plastic deformation. However, at higher temperatures creep deformation becomes more significant in the alloy, which acts to lower the applied stress through stress relaxation, since the test is under strain control. Under an IP TMF cycle, with creep dominating at the peak strain, this stress relaxation acts to decrease the mean stress throughout the test. Conversely in a $-180^{\circ}$ OOP test, creep is most prevalent at the minimum strain.

Clearly for a series of tests performed at different strain ranges, this approach is overly simplistic in the presence of varying peak stresses and stress ranges. For example a $-180^{\circ}$ OOP test with a high strain range would not be expected to show a longer fatigue life than an IP test with a low strain range, simply because it has a lower value of mean stress. However, for the current evaluation of the effect of phase angle on fatigue life, the method can be instructive for a series of experiments where a constant strain range is utilised.

Following initial plasticity on loading, as demonstrated in Fig. 3, similar stress ranges occur across the range of phase angles, enabling the effect of mean stress to be more clearly evaluated. Figure 5 shows a comparison of mean stress against normalised cycles to failure for the range of tests performed at $\Delta \varepsilon_{\text {mech }}=1 \%$. The data is normalised by the number of fatigue cycles to failure for an isothermal test, performed at the peak temperature $\left(700^{\circ} \mathrm{C}\right)$ of the TMF cycle and clearly demonstrates the effects of mean stress, with $\mathrm{x}$-axis values $<1$ showing a detriment to life, and values $>1$ showing an extension of life. With an $\mathrm{R}^{2}$ value of 0.81 it is apparent that a relationship exists between TMF life and mean stress. However, it should again be emphasised that this relationship exists only for specimens with a comparable stabilised stress range, brought about in these experiments by the application of a constant applied strain range of $1 \%$ throughout the tests. In the case of extrapolation to a varying applied strain range (and hence stabilised stress range) the relationship holds little value because of the tendency of stabilised stress range to become the dominant factor.

Typically, more holistic fatigue lifing approaches will be either strain range $[5,6,13]$ or stress range [14] based.

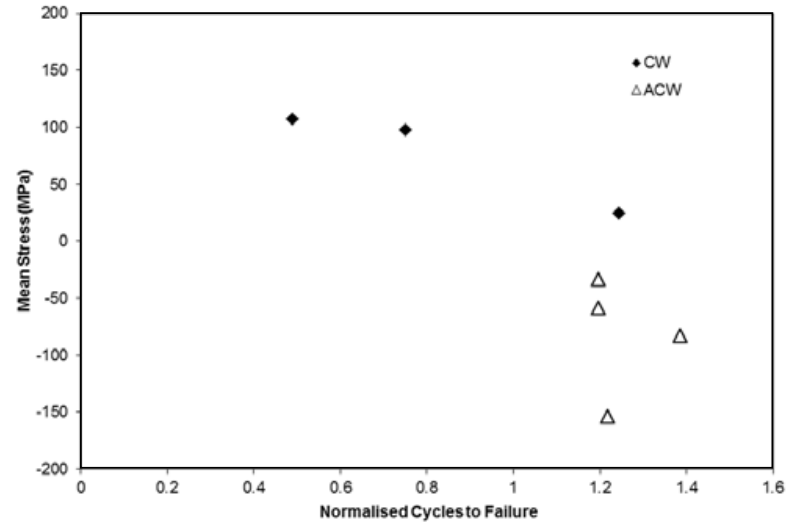

Figure 5. Mean stress vs. cycles to failure for varying phase angle tests at $\Delta \varepsilon$ of $1 \%$.

In the current work a strong correlation between stabilised stress range and TMF life in the IP and $-180^{\circ}$ OOP experiments indicates that a Basquin [14] type approach could potentially form the basis of a model which is capable of TMF life prediction and extrapolation across a range of phase angles.

As mentioned previously however, TMF experiments are costly, difficult and time consuming and it is preferable that predictions can be made based on isothermal data. However, as indicated in the literature [15], simple "worst case scenario" and generalised fatigue life reduction factors fail to capture the complexities of the effect of phase angle, potentially leading to overly conservative life predictions or even premature component failures. In the present work, efforts have been made to affect a creep and strain controlled fatigue prediction based on TMF hysteresis loops which have been derived from a database of isothermal RR1000 test results. SC03 is a non-linear finite element analysis program which utilises the Mroz multilayer plasticity model to predict cyclic deformation based on stress-strain data obtained from isothermal testing [16]. The wide range of input data used to produce the deformation model for RR 1000 provides confidence in its ability to predict conditions outside of the envelope of this original data. It also allows for an iterative approach over both temperature and strain conditions by which the replication of TMF stress-strain hysteresis loops can be achieved.

Examples of loops produced by $\mathrm{SC0} 3$ are shown in Fig. 6 and compared with experimental data generated under TMF loading conditions. It can be seen that peak stress, minimum stress and loop shape are all captured well by the model, providing confidence in its ability to extrapolate to alternative phase angles and produce a model which can provide TMF predictions over a wide range of phase angles when used in association with predicted stresses from SC03 to estimate TMF test failure lives. As described earlier a strong relationship exists between the stabilised stress range and the fatigue life, and as such Basquin's law [14] has been applied to derive a predictive equation for the experiments.

In order to prove the effectiveness of the technique, the predictive equation has been derived based purely on IP experiments. Stress-strain hysteresis loops were then 




(a)

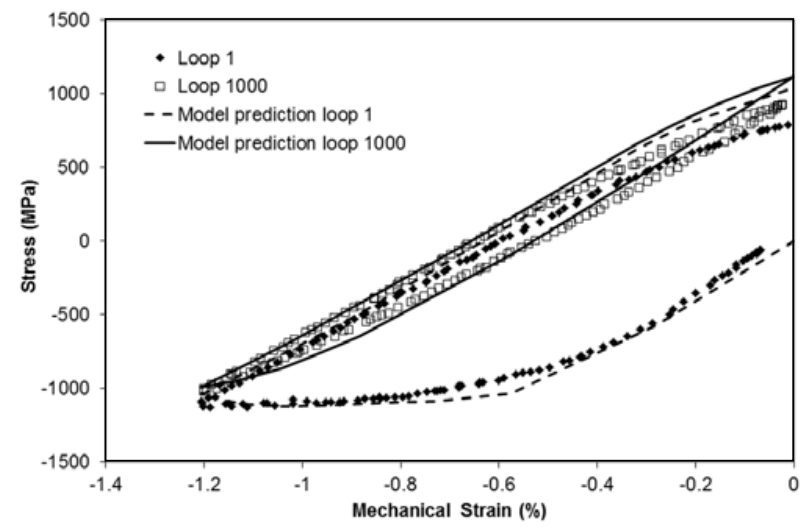

(b)

Figure 6. Modelling of TMF loops (a) IP $\Delta \varepsilon$ of $0.8 \%$, (b) $-180^{\circ} \mathrm{OOP} \Delta \varepsilon$ of $1.2 \%$.

produced for each of the remaining tested conditions $(4 \times$ $\left.180^{\circ} \mathrm{OOP}, 45^{\circ} \mathrm{CW}, 90^{\circ} \mathrm{CW}, 90^{\circ} \mathrm{ACW}, 135^{\circ} \mathrm{ACW}\right)$ and predictions made of TMF life using the Basquin approach [15]. The equation takes the form

$$
\frac{\Delta \sigma}{2}=\sigma_{f}^{\prime}\left(2 N_{f}\right)^{b}
$$

where $\Delta \sigma$ is the stress range, $\mathrm{N}_{\mathrm{f}}$ is the number of TMF cycles to failure again normalised by the fatigue life of an isothermal test at $\Delta \varepsilon=1 \%$ at peak cycle temperature $\left(700{ }^{\circ} \mathrm{C}\right)$, for consistency. The constants were optimised at $\sigma_{f}^{\prime}=989.5, b=-0.0818$.

Figure 7 shows the predictions of phase angles between 0 and $-180^{\circ}$ using the above equation. It is clear that reasonable predictions are obtained for all of the data but the method has the tendency to slightly u nderpredict the results. This may be a result of differences in the crack propagation life of the IP and $-180^{\circ} \mathrm{OOP}$ tests brought about by the difference in temperature at peak stress. Furthermore it could be argued that when considering the range of phase angle tests performed at $\Delta \varepsilon_{\text {mech }}=1 \%$ the method fails to capture differences between the phase angles and certainly does not separate the results in the manner in which the mean stress approach did. It should be noted that only a factor of 2 difference is observed in the failure life of these experiments, and for this reason it is difficult to draw strong conclusions. Furthermore, it is also necessary to consider that due to only limited creep strain accumulation at the peak cycle temperature, the

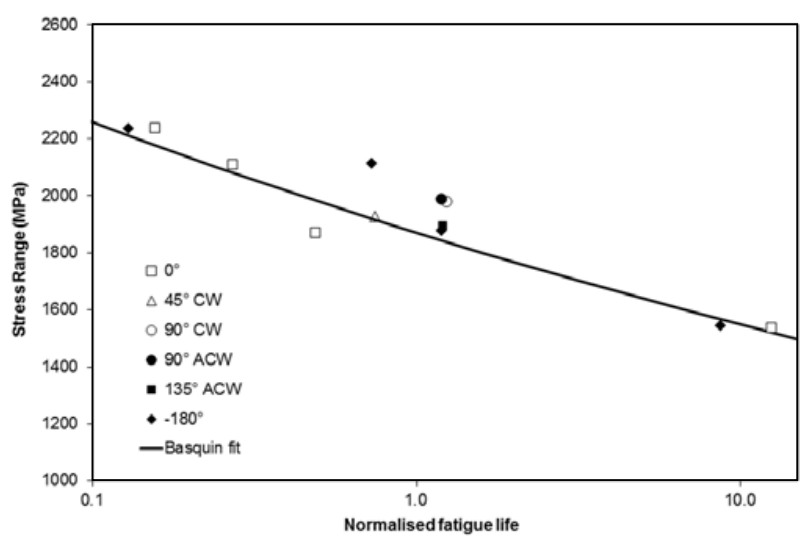

Figure 7. Basquin fit predictions of TMF behaviour.

accumulation of significant mean stresses does not occur in the current tests. Future approaches for higher peak cycle temperatures may be well advised to consider either an adaptation of the Basquin approach to also consider mean stress, or the application of an approach based on both stress range and mean stress.

Both of the approaches detailed however, consider only a total life philosophy. Previous work [17] has indicated that the ranking of IP, OOP and isothermal fatigue tests may prove difficult for short TMF life experiments when using only methods such as Basquin [14] or MansonCoffin [5,6]. Indeed the ordering of these test results may prove counterintuitive to the ordering seen for longer life experiments. As such it is recommended to further investigate the length of the crack propagation phase within the current series of tests, so that the ratio of crack initiation/propagation can be considered and understood.

\section{Conclusions}

Since the recognition of TMF as a significant life limiting factor engineers have desired a methodology by which TMF behaviour can be accurately described by extrapolation of isothermal fatigue data, so that the wide range of possible parameters can be predicted without the need for large, expensive, protracted test programmes. The current work has described three methods by which predictions can be made. Under test conditions where a constant strain range is utilised across phase angles, it is shown that mean stress is the dominant factor and that a relationship exists between mean stress and fatigue life. This methodology, however, soon falters as the mechanical strain range is varied because of the resultant changes in the stabilised stress range. In this case it is demonstrated that a simple Basquin type equation can be effective as a life prediction technique, albeit for a range of TMF data which shows only short lives. It is acknowledged that such an approach is unlikely to extrapolate well to longer fatigue lives, because of the proportionate difference in the ratio of crack initiation/propagation that is likely to occur. As such, it is concluded that a combination of a Basquin type approach based on longer term TMF data, supplemented by a crack propagation phase is the most likely method to provide accurate predictions over a range of test conditions. 
The current research was funded under the EPSRC RollsRoyce Strategic Partnership in Structural Metallic Systems for Gas Turbines (grants EP/H500383/1 and EP/H022309/1). The provision of materials and supporting information from RollsRoyce plc is gratefully acknowledged as is the contribution of Dr. Svjetlana Stekovic.

\section{References}

[1] H.J. Christ, A. Jung, H.J. Maier, R. Teteruk, Thermomechanical fatigue - Damage mechanisms and mechanism-based life prediction methods. Sadhana, 28, 147-165, (2003)

[2] H. Sehitoglu, H.J. Maier, Thermo-mechanical Fatigue Behaviour of Materials: ASTM STP, 3, 1371. (2000)

[3] M. Sakaguchi, M. Okazaki, Thermo-mechanical and low cycle fatigues of single crystal $\mathrm{Ni}$ base superalloys; Importance of microstructure for life prediction. JSME Journal, 49, 345-354 (2006)

[4] J.J Pernot, T. Nicholas, S. Mall, Modelling thermomechanical fatigue crack growth rates in Ti-24Al11Nb. Int J Fatigue, 16, 111-112 (1994)

[5] L.F. Coffin, Fatigue at Elevated Temperatures. ASTM STP, 520, 744-782 (1973)

[6] S.S Manson, Fatigue - A complex subject - Some simple approximations. Exp Mech, 5, 193-226 (1965)

[7] J.X. Zhang, H. Harada, Y. Koizumi, T. Kobayashi, Crack appearance of single-crystal nickel-base superalloys after thermomechanical fatigue failure. Scripta Mater, 61, 1105-1108 (2009)

[8] H. Kang, Y. Lee, J. Chen, J.D. Fan, Thermomechanical fatigue damage model for variable temperature and loading amplitude conditions. Int $\mathbf{J}$ Fatigue, 29, 1797-1802 (2007)

[9] M. Okazaki, T. Koizumi, Relationship of crack growth between thermal-mechanical and isothermal low-cycle fatigue at elevated temperatures. J Eng Mater T, 109, 114-118 (1987)

[10] E.H. Jordan, G.J. Meyers, Fracture mechanics applied to nonisothermal fatigue crack growth. Eng Fract Mech, 23, 345-358 (1986)

[11] ISO 12111:2011 - Metallic materials - Fatigue testing - Strain-controlled thermo mechanical fatigue testing method. BSI Standards Publication (2011)

[12] P. Hahner, C. Rinaldi, V. Bicego, E. Affeldt, T. Brendel, H. Andersson, T. Beck, H. Klingelhoffer, H. Kuhn, A. Koster, M. Loveday, M. Marchionni, C. Rae, Research and development into a European code-of practice for strain-controlled thermomechanical fatigue testing. Int J Fatigue, 30, 372-381 (2007)

[13] K. Walker, Effects of environment and complex loading history on fatigue life. ASTM STP, 462, 114. (1970)

[14] O.H. Basquin, The exponential law of endurance tests. ASTM, 10, 625-630 (1919)

[15] W.J. Evans, J.E. Screech, S.J. Williams, Thermomechanical fatigue and fracture of INCO718. Int J Fatigue, 30, 257-267 (2008)

[16] Z. Mroz, An attempt to describe the behaviour of metals under cyclic loads using a more general workhardening model. Acta Mech, 7, 199-212 (1967)

[17] R.J. Lancaster, M.T. Whittaker, S.J. Williams, A review of thermo-mechanical fatigue behaviour in polycrystalline nickel superalloys for turbine disc applications. Mater High Temp, 30, 2-12 (2013) 